\title{
Assessment Of Peripapillary Retinal Nerve Fiber Layer Thickness Post Pars Plana Vitrectomy By Ocular Coherence Tomograghy
}

Prof. Dr. Hossam Eldein Mohamed Khalil, Dr. Mostafa Abdul Naby Saeed, Dr. Sherif Momtaz Hegazy \& Mostafa Mahmoud Eid Mahmoud*

Ophthalmology Department, Faculty of Medicine, Beni-Suef University

And Research Institute of Ophthalmology

\begin{abstract}
:
Background: Modifications of the inner retinal layer is a frequent observation following pars plana vitectomy. Aim of the Work: To assess early changes of the thickness of Retinal Nerve Fiber Layer after pars plana vitrectomy by ocular coherence tomography. Patients and Methods: This is a non-interventional observational study, in which 30 eyes of 30 patients underwent Examination of Retinal Nerve Fiber Layer after Pars Plana Vitrectomy by OCT. Cases were performed at the Ophthalmology department of Beni-Suef University`s Hospital and Research Institute of Ophthalmology. Results: In the current study we found by using the OCT, there were no changes in the peri-papillary retinal nerve fiber layer that can sound statistically. PPV had no effect on RNFL because of patient Age or Gender or ether eye status like the underlaying pathology, axial length of the eye except at some quadrants of RNFL. Conclusion: Despite RNFL showing changes following vitrectomy, they showed no statistical significance.
\end{abstract}

Keywords: Ocular Coherence Tomograghy - Pars Plana Vitrectomy - Ganglion Cell Complex.

\section{Introduction}

The Ganglion cell complex (GCC) has been defined as a region including the nerve fiber layer (NFL), the ganglion cell layer, and the inner plexiform layer. It's clinical importance is to evaluate the morphology of the inner retinal layers. NFL is located between Internal Limiting Membrane and the ganglion cell layer of the retina. The NFL develops from Retinal Ganglion Cells axons 
converging on the disc to form bundles. The thickness of the NFL bundles is greater at the disc and decreases towards periphery ${ }^{(\mathbf{1})}$.

Inner retina layer modifications after pars plana vitrectomy have been studied extensively in recent years ${ }^{(2)}$. Some studies have reported NFL thickening after PPVsecondary to mechanical insult due to direct mechanical traction on the papillomacular bundle resulting in tenting and elevation of nerve fibers or rebound edema of the inner retinal layers in the first postoperative week ${ }^{(1)}$. Other studies have reported thinning with possible mechanisms include NFL dehydration during fluid-air exchange, dye-related toxicity, mechanical injury or increased intraocular pressure (IOP) (2).

IOP elevation is reported after PPV and silicone oil (SO) injection, with an incidence ranging from $3 \%$ to $40 \%$ of cases during and after vitrectomy. $20 \%$ of these patients had no history of glaucoma or ocular hypertension and most occurred in the first month after PPV ${ }^{(3)}$.

Involved mechanisms of the IOP elevation include open angle glaucoma due to the blockage of trabecular meshwork by oil, intraocular inflammation induced by the surgery, response to the steroid topical therapy or closed angle glaucoma ${ }^{(3)}$.

There are several technologies that have been used for measuring the NFL, these include scanning laser polarimetry, confocal scanning laser ophthalmoscopy, and ocular coherence tomography (OCT). The development of spectral domain optical coherence tomography (SD-OCT) enabled morphological study of different retinal layers possible with a high level of detail ${ }^{(\mathbf{1})}$.

OCT is now the most commonly used optical imaging technique for the measurement of the NFL, and seems likely to supersede other technologies. It uses lowcoherence interferometry to calculate RNFL thickness by measuring the interval of light back scattered from the retina and a reference mirror $^{(3)}$

\section{Aim Of The Work}

To assess early changes of the thickness of Retinal Nerve Fiber Layer after pars plana vitrectomy by ocular coherence tomography.

\section{Patients And Methods}

\section{Patients:}

\section{a. Sample Size:}

A test was used to estimate the sample size. A sample size of 30 participants achieved $80 \%$ power to detect an effect size (W) of 0.40 with a significance level (alpha) of 0.05 .

\section{By using this formula:}


$\mathbf{n}=\mathbf{N} * \mathbf{X} /(\mathbf{X}+\mathbf{N}-\mathbf{1})$ Where, $\mathbf{X}=$ $\mathbf{Z}_{\mathbf{a} / \mathbf{2}}{ }^{2} * \mathbf{p} *(\mathbf{1}-\mathbf{p}) / \mathbf{M O E}$, and $\mathbf{Z}_{\mathbf{\alpha} / \mathbf{2}}$ is the critical value of the Normal distribution at $\alpha / 2$ (e.g. for a confidence level of $95 \%, \alpha$ is 0.05 and the critical value is 1.96), MOE is the margin of error, $\mathbf{P}$ is the sample proportion, and $\mathbf{N}$ is the population size.

\section{b. Type of the study:}

This is a non-interventional observational study, in which (30) patients underwent examination of Retinal Nerve Fiber Layer after Pars Plana Vitrectomy by OCT.

\section{c. Site of the study:}

Ophthalmology department of BeniSuef University`s Hospital and Research Institute of Ophthalmology.

Date and period of the Study:

This study was conducted between July 2019 through September 2019.

\section{d. Ethical considerations:}

Patients were enrolled consecutively after the approval of the Ethical Committee of the Beni-Suef University. A written consent was signed by all participants after discussing the procedure, alternative treatment Plans, follow up schedules and possible benefits and risks.

\section{e. Inclusion criteria:}

- Patients who had Pars Plana Vitrectomy
- Age group between 18-70 years

f. Exclusion criteria:

1. Any ocular trauma.

2. Previous ocular surgery.

3. Significant media opacity after operation.

4. History of inflammation within eye as uveitis.

5. Age extremities above 70 years or below 18 years.

6. Glaucoma patients.

7.

\section{Methods:}

\section{All patients underwent:}

1. Full history taking.

2. Full ophthalmic examination. (Visual acuity, IOP measurement, Slit lamp and Fundus examination)

3. All patients underwent peripapillary NFL average measurement by Spectral Domain OCT (SD-OCT) using Heidelberg OCT II (Heidelberg Engineering, Dossenheim, Germany) at 1 week, 1 month \& 3months after vitrectomy.

\section{Statistical Analysis}

Recorded data were analyzed using the Statistical package for Social Sciences, version 20.0 (SPSS Inc., Chicago, Illinois, USA). Quantitative data were expressed as mean \pm standard deviation (SD). Qualitative 
data were expressed as frequency and percentage.

\section{The following tests were done:}

Independent-samples t-test of significance was used when comparing between two means. Paired sample t-test of significance was used when comparing between related samples. A one-way analysis of variance (ANOVA) was used when comparing between more than two means. Pearson's correlation coefficient (r) test was used to assess the degree of association between two sets of variables. The confidence interval was set to $95 \%$ and the margin of error accepted was set to $5 \%$. So, the p-value was considered significant as the following:

\section{Probability (P-value)}

- P-value $<0.05$ was considered significant.

- P-value <0.001 was considered as highly significant.

- P-value >0.05 was considered insignificant.

\section{Results:}

\section{Preoperative Retinal Nerve Fiber Layer} Analysis:

The 30 patients comprised 21 men and 9 women. Mean patient age was 53.00 \pm 12.21 years (mean $\pm \mathrm{SD}$; range, 30-70 years).

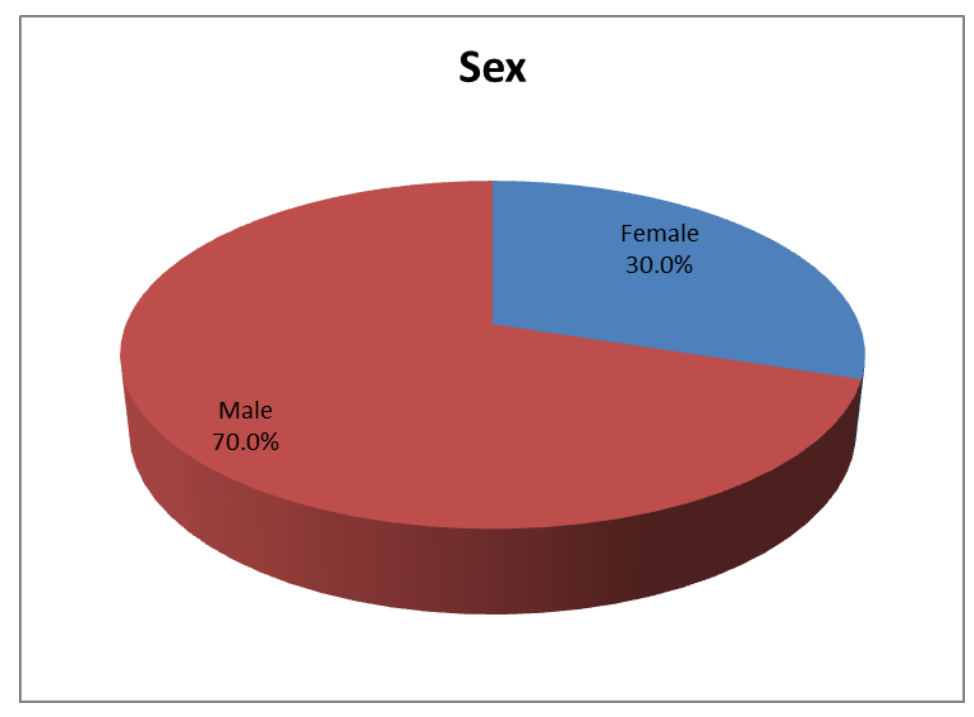

Fig. (1): Pie chart sex distribution of the study group.

The mean axial length of the vitrectomized eyes (mean \pm SD) was $25.07 \pm 1.72 \mathrm{~mm}$, the ranges of axial length were $22-28 \mathrm{~mm}$. 


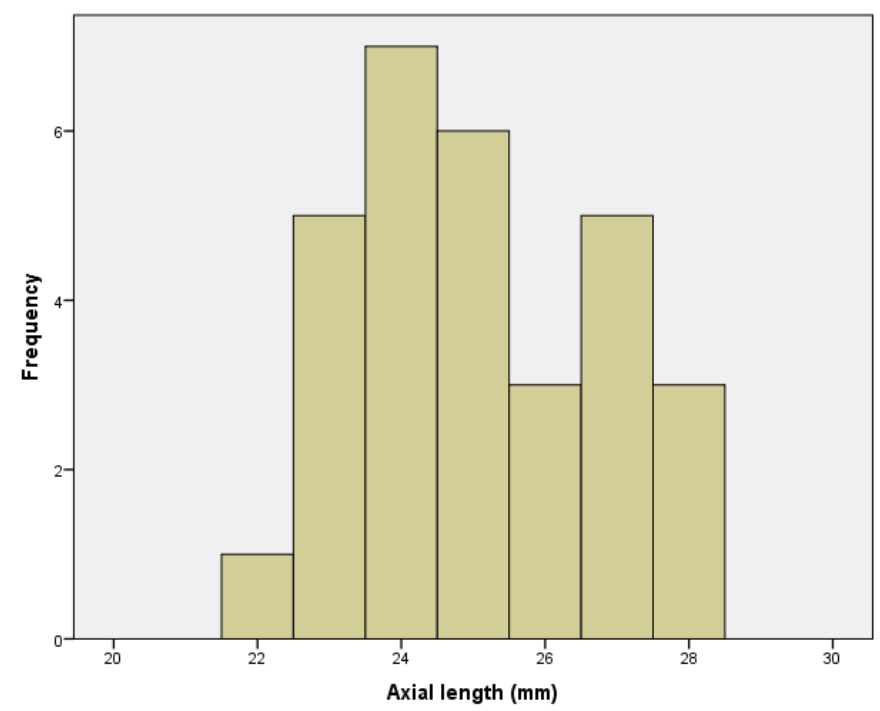

Fig. (2): Histogram age distribution of the study group.

Nineteen eyes underwent vitrectomy combined with cataract surgery. Ten cases underwent stain assisted ILM peeling; PFC was used in ten cases. Endolaser, silicone endotamponade and triamcinolone acetanoide was used for all cases.

In Seventeen cases; retinal detachment was the original pathology while in thirteen cases was diabetic retinopathy.

Thirteen patients have no systemic disease while eight patients were diabetic and nine patients suffered from both diabetes and hypertension.

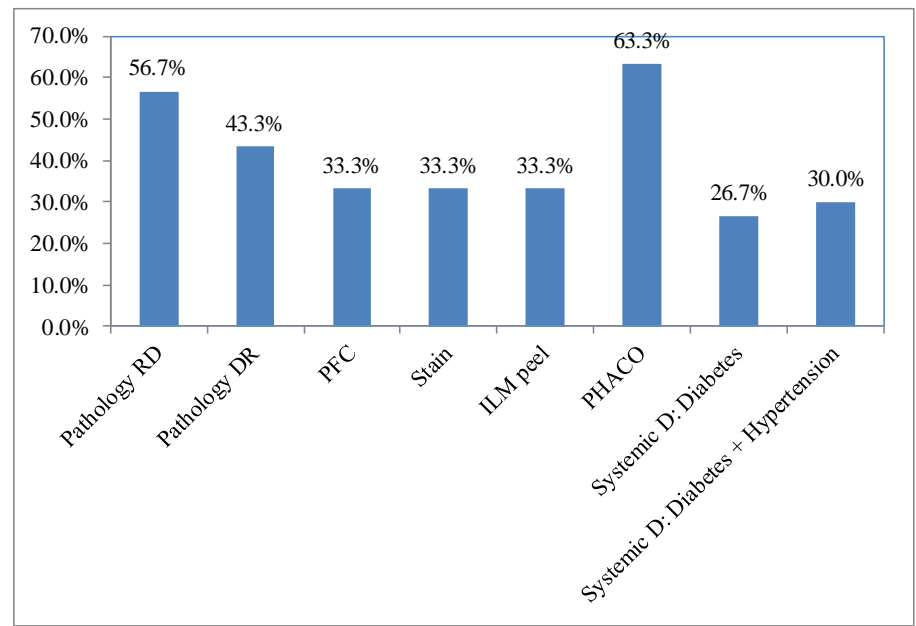

Fig. (3): Bar chart Pathology, PFC, Stain, ILM peel, systemic diseases distribution of the study group. 


\section{Changes in RNFL Thickness after Vitrectomy:}

The regional RNFL thickness in the superior nasal, superior temporal, inferior nasal, inferior temporal, temporal, and nasal quadrants did not differ significantly between first week, one month and 3 months follow up.

Mean RNFL thickness showed no significant change during follow up: at the first week

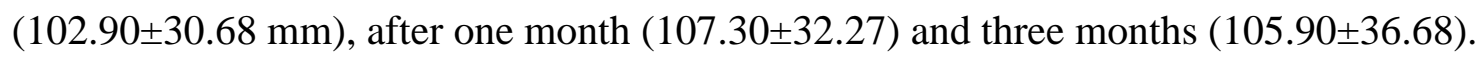

Table (1): The extent of the difference over the periods through NFL G in the study group

\begin{tabular}{|c|c|c|c|c|c|}
\hline \multirow{2}{*}{ Period } & \multicolumn{2}{|c|}{ NFL G } & \multicolumn{3}{c|}{ Paired Sample t-test } \\
\cline { 2 - 6 } & Range & Mean \pm SD & Mean Diff. & t-test & p-value \\
\hline After 1wk. & $50-175$ & $102.90 \pm 30.68$ & & & \\
\hline After 1m. & $53-191$ & $107.30 \pm 32.27$ & 4.40 & -0.740 & 0.466 \\
\hline After 3m. & $50-207$ & $105.90 \pm 36.68$ & 3.00 & -0.410 & 0.685 \\
\hline
\end{tabular}

p-value $>0.05 \mathrm{NS}$

Mean RNFL thickness (mean \pm SD) at the first week was $112.07 \pm 41.02 \mu \mathrm{m}$ in the superior nasal quadrant, $120.13 \pm 44.92 \mathrm{~mm}$ superior temporal quadrant, $117.30 \pm 47.53 \mathrm{~mm}$ in the inferior nasal quadrant, $142.20 \pm 51.41$ inferior temporal quadrant, $90.57 \pm 30.06 \mathrm{~mm}$ in the temporal quadrant, and $92.53 \pm 31.43 \mathrm{~mm}$ in the nasal quadrant.

Mean RNFL thickness of the at the first month was $111.47 \pm 50.97 \mathrm{~mm}$ in the superior nasal quadrant, $124.90 \pm 54.08$ superior temporal quadrant, $120.10 \pm 43.69 \mathrm{~mm}$ in the inferior nasal quadrant, $142.30 \pm 52.32$ inferior temporal quadrant, $104.63 \pm 46.20 \mathrm{~mm}$ in the temporal quadrant, and $88.43 \pm 28.80 \mathrm{~mm}$ in the nasal quadrant.

Mean RNFL thickness of the at the third month was $110.70 \pm 56.86 \mathrm{~mm}$ in the superior nasal quadrant, $131.70 \pm 66.01$ superior temporal quadrant, $112.93 \pm 38.92 \mathrm{~mm}$ in the inferior nasal quadrant, $129.87 \pm 51.66$ inferior temporal quadrant, $107.57 \pm 62.23 \mathrm{~mm}$ in the temporal quadrant, and $88.87 \pm 36.78 \mathrm{~mm}$ in the nasal quadrant. 
Table (2): The extent of the difference over the periods through NFL $\mathrm{N}$ in the study group

\begin{tabular}{|c|c|c|c|c|c|}
\hline \multirow{2}{*}{ Period } & \multicolumn{2}{|c|}{ NFL N } & \multicolumn{2}{c|}{ Paired Sample t-test } \\
\cline { 2 - 6 } & Range & Mean \pm SD & Mean Diff. & t-test & p-value \\
\hline After 1wk. & $35-157$ & $92.53 \pm 31.43$ & & & \\
\hline After 1m. & $38-182$ & $88.43 \pm 28.80$ & -4.10 & 0.673 & 0.506 \\
\hline After 3m. & $28-183$ & $88.87 \pm 36.78$ & -3.70 & 0.509 & 0.615 \\
\hline
\end{tabular}

p-value $>0.05 \mathrm{NS}$

Table (3): The extent of the difference over the periods through NFL $\mathrm{T}$ in the study group

\begin{tabular}{|c|c|c|c|c|c|}
\hline \multirow{2}{*}{ Period } & \multicolumn{2}{|c|}{ NFL T } & \multicolumn{2}{c|}{ Paired Sample t-test } \\
\cline { 2 - 6 } & Range & Mean \pm SD & Mean Diff. & t-test & p-value \\
\hline After 1wk. & $39-159$ & $90.57 \pm 30.06$ & & & \\
\hline After 1m. & $49-289$ & $104.63 \pm 46.20$ & 14.10 & -1.682 & 0.103 \\
\hline After 3m. & $40-265$ & $107.57 \pm 62.23$ & 17.00 & -1.541 & 0.134 \\
\hline
\end{tabular}

p-value $>0.05 \mathrm{NS}$

Table (4): The extent of the difference over the periods through NFL SN in the study group

\begin{tabular}{|c|c|c|c|c|c|}
\hline \multirow{2}{*}{ Period } & \multicolumn{2}{|c|}{ NFL SN } & \multicolumn{2}{c|}{ Paired Sample t-test } \\
\cline { 2 - 6 } & Range & Mean \pm SD & Mean Diff. & t-test & p-value \\
\hline After 1wk. & $43-208$ & $112.07 \pm 41.02$ & & & \\
\hline After 1m. & $22-315$ & $111.47 \pm 50.97$ & -0.60 & 0.069 & 0.946 \\
\hline After 3m. & $20-251$ & $110.70 \pm 56.86$ & -1.40 & 0.132 & 0.896 \\
\hline
\end{tabular}

p-value $>0.05 \mathrm{NS}$

Table (5): The extent of the difference over the periods through NFL ST in the study group

\begin{tabular}{|c|c|c|c|c|c|}
\hline \multirow{2}{*}{ Period } & \multicolumn{2}{|c|}{ NFL ST } & \multicolumn{2}{c|}{ Paired Sample t-test } \\
\cline { 2 - 6 } & Range & Mean \pm SD & Mean Diff. & t-test & p-value \\
\hline After 1wk. & $14-200$ & $120.13 \pm 44.92$ & & & \\
\hline After 1m. & $24-242$ & $124.90 \pm 54.08$ & 4.80 & -0.407 & 0.687 \\
\hline After 3m. & $24-288$ & $131.70 \pm 66.01$ & 11.60 & -0.804 & 0.428 \\
\hline
\end{tabular}


Table (6): The extent of the difference over the periods through NFL IN in the study group

\begin{tabular}{|c|c|c|c|c|c|}
\hline \multirow{2}{*}{ Period } & \multicolumn{2}{|c|}{ NFL IN } & \multicolumn{2}{c|}{ Paired Sample t-test } \\
\cline { 2 - 6 } & Range & Mean \pm SD & Mean Diff. & t-test & p-value \\
\hline After 1wk. & $28-280$ & $117.30 \pm 47.53$ & & & \\
\hline After 1m. & $59-241$ & $120.10 \pm 43.69$ & 2.80 & -0.391 & 0.699 \\
\hline After 3m. & $53-187$ & $112.93 \pm 38.92$ & -4.40 & 0.589 & 0.560 \\
\hline
\end{tabular}

p-value $>0.05 \mathrm{NS}$

Table (7): The extent of the difference over the periods through NFL IT in the study group

\begin{tabular}{|c|c|c|c|c|c|}
\hline \multirow{2}{*}{ Period } & \multicolumn{2}{|c|}{ NFL IT } & \multicolumn{2}{c|}{ Paired Sample t-test } \\
\cline { 2 - 6 } & Range & Mean \pm SD & Mean Diff. & t-test & p-value \\
\hline After 1wk. & $28-240$ & $142.20 \pm 51.41$ & & & \\
\hline After 1m. & $56-259$ & $142.30 \pm 52.32$ & 0.10 & -0.012 & 0.990 \\
\hline After 3m. & $60-269$ & $129.87 \pm 51.66$ & -12.30 & 1.244 & 0.224 \\
\hline
\end{tabular}

p-value $>0.05 \mathrm{NS}$

\section{Changes in Retinal Nerve Fiber Layer Thickness According to the Eye and patient status:}

The RNFL thickness in each quadrant was not significantly different by patient gender (table 8) or age group (table 9). However, axial length of the eye was significantly different at average, nasal and superior temporal at 3 months follow up visit (table 10).

Table (8): Comparison between female and male according to all data in the study group

\begin{tabular}{|c|c|c|c|c|c|c|}
\hline \multirow{2}{*}{ Parameters } & \multicolumn{2}{|c|}{ Female } & \multicolumn{2}{c|}{ Male } & \multicolumn{2}{c|}{ t-test } \\
\cline { 2 - 7 } & Mean & \pm SD & Mean & \pm SD & T & p-value \\
\hline NFL G Change 1w after 1m. & 3.30 & 44.03 & 14.88 & 49.41 & 0.368 & 0.549 \\
\hline NFL G Change 1w after 3m. & -3.97 & 53.33 & 17.15 & 46.48 & 1.193 & 0.284 \\
\hline NFL N Change 1w after 1m. & -7.65 & 36.56 & 7.53 & 40.36 & 0.939 & 0.341 \\
\hline NFL N Change 1w after 3m. & -14.66 & 47.03 & 11.88 & 50.34 & 1.817 & 0.188 \\
\hline NFLT Change 1w after 1m. & 9.66 & 42.65 & 30.96 & 78.33 & 0.583 & 0.452 \\
\hline NFLT Change 1w after 3m. & 0.72 & 47.07 & 37.04 & 87.49 & 1.363 & 0.253 \\
\hline NFL SN Change 1w after 1m. & -17.58 & 24.29 & 17.94 & 70.15 & 2.157 & 0.153 \\
\hline
\end{tabular}




\begin{tabular}{|l|c|c|c|c|c|c|}
\hline NFL SN Change 1w after 3m. & -10.74 & 52.37 & 15.55 & 70.23 & 1.011 & 0.323 \\
\hline NFL ST Change 1w after 1m. & 13.90 & 122.64 & 64.32 & 202.86 & 0.475 & 0.496 \\
\hline NFL ST Change 1w after 3m. & 7.80 & 108.41 & 92.56 & 291.32 & 0.708 & 0.407 \\
\hline NFL IN Change 1w after 1m. & 16.25 & 75.96 & 13.57 & 53.44 & 0.012 & 0.913 \\
\hline NFL IN Change 1w after 3m. & 5.95 & 61.72 & 8.93 & 51.40 & 0.019 & 0.892 \\
\hline NFL IT Change 1w after 1m. & 27.71 & 118.27 & 13.00 & 76.49 & 0.167 & 0.686 \\
\hline NFL IT Change 1w after 3m. & -3.64 & 95.50 & 12.23 & 64.87 & 0.283 & 0.599 \\
\hline
\end{tabular}

Table (9): Correlations between ages with all data, using Pearson Correlation Coefficient in the study group

\begin{tabular}{|l|c|c|}
\hline \multirow{2}{*}{ Parameters } & \multicolumn{2}{|c|}{ Age (years) } \\
\cline { 2 - 3 } & R & p-value \\
\hline NFL G Change 1w after 1m. & 0.085 & 0.657 \\
\hline NFL G Change 1w after 3m. & 0.017 & 0.930 \\
\hline NFL N Change 1w after 1m. & -0.163 & 0.388 \\
\hline NFL N Change 1w after 3m. & -0.173 & 0.362 \\
\hline NFLT Change 1w after 1m. & -0.078 & 0.681 \\
\hline NFLT Change 1w after 3m. & 0.042 & 0.825 \\
\hline NFL SN Change 1w after 1m. & 0.196 & 0.298 \\
\hline NFL SN Change 1w after 3m. & 0.010 & 0.958 \\
\hline NFL ST Change 1w after 1m. & 0.216 & 0.251 \\
\hline NFL ST Change 1w after 3m. & 0.177 & 0.349 \\
\hline NFL IN Change 1w after 1m. & -0.111 & 0.559 \\
\hline NFL IN Change 1w after 3m. & -0.244 & 0.194 \\
\hline NFL IT Change 1w after 1m. & 0.089 & 0.639 \\
\hline NFL IT Change 1w after 3m. & 0.028 & 0.884 \\
\hline
\end{tabular}


Table (10): Correlation between axial lengths with all data, using Pearson Correlation Coefficient in the study group

\begin{tabular}{|c|c|c|}
\hline \multirow{2}{*}{ Parameters } & \multicolumn{2}{|c|}{ Axial length (mm) } \\
\cline { 2 - 3 } & R & p-value \\
\hline NFL G Change 1w after 1m. & 0.321 & 0.084 \\
\hline NFL G Change 1w after 3m. & $.373^{*}$ & 0.042 \\
\hline NFL N Change 1w after 1m. & $.456^{*}$ & 0.011 \\
\hline NFL N Change 1w after 3m. & $.409^{*}$ & 0.025 \\
\hline NFLT Change 1w after 1m. & 0.313 & 0.092 \\
\hline NFLT Change 1w after 3m. & 0.289 & 0.122 \\
\hline NFL SN Change 1w after 1m. & 0.261 & 0.164 \\
\hline NFL SN Change 1w after 3m. & 0.354 & 0.055 \\
\hline NFL ST Change 1w after 1m. & $.374^{*}$ & 0.042 \\
\hline NFL ST Change 1w after 3m. & $.382^{*}$ & 0.037 \\
\hline NFL IN Change 1w after 1m. & 0.255 & 0.173 \\
\hline NFL IN Change 1w after 3m. & 0.340 & 0.066 \\
\hline NFL IT Change 1w after 1m. & 0.145 & 0.444 \\
\hline NFL IT Change 1w after 3m. & 0.229 & 0.223 \\
\hline
\end{tabular}

\section{Changes in Retinal Nerve Fiber Layer Thickness According to the pathology:}

The RNFL thickness in each quadrant was not significantly different by pathology group.

Table (11): Comparison between RD and DR according to all data in the study group

\begin{tabular}{|c|c|c|c|c|c|c|}
\hline \multirow{2}{*}{ Parameters } & \multicolumn{2}{|c|}{ RD } & \multicolumn{2}{c|}{ DR } & \multicolumn{2}{c|}{ t-test } \\
\cline { 2 - 7 } & Mean & \pm SD & Mean & \pm SD & T & p-value \\
\hline NFL G Change 1w after 1m. & 19.00 & 52.64 & 1.48 & 39.35 & 1.005 & 0.325 \\
\hline NFL G Change 1w after 3m. & 19.24 & 53.37 & -0.20 & 41.25 & 1.181 & 0.286 \\
\hline NFL N Change 1w after 1m. & 10.75 & 42.94 & -7.19 & 32.73 & 1.567 & 0.221 \\
\hline NFL N Change 1w after 3m. & 13.56 & 58.34 & -8.68 & 35.02 & 1.474 & 0.235 \\
\hline NFLT Change 1w after 1m. & 34.08 & 88.17 & 12.13 & 31.90 & 0.727 & 0.401 \\
\hline NFLT Change 1w after 3m. & 34.80 & 96.10 & 14.82 & 48.66 & 0.468 & 0.500 \\
\hline
\end{tabular}




\begin{tabular}{|l|c|c|c|c|c|c|}
\hline NFL SN Change 1w after 1m. & 11.12 & 75.71 & 2.28 & 39.59 & 0.146 & 0.705 \\
\hline NFL SN Change 1w after 3m. & 18.34 & 79.94 & -6.31 & 38.94 & 1.041 & 0.316 \\
\hline NFL ST Change 1w after 1m. & 75.62 & 224.77 & 14.64 & 101.50 & 0.823 & 0.372 \\
\hline NFL ST Change 1w after 3m. & 105.91 & 322.13 & 16.43 & 98.90 & 0.929 & 0.343 \\
\hline NFL IN Change 1w after 1m. & 21.96 & 55.75 & 4.47 & 65.41 & 0.624 & 0.436 \\
\hline NFL IN Change 1w after 3m. & 17.46 & 51.81 & -4.29 & 55.48 & 1.221 & 0.279 \\
\hline NFL IT Change 1w after 1m. & 15.61 & 84.38 & 19.77 & 98.42 & 0.015 & 0.902 \\
\hline NFL IT Change 1w after 3m. & 9.26 & 74.09 & 5.12 & 76.78 & 0.022 & 0.882 \\
\hline
\end{tabular}

\section{Changes in Retinal Nerve Fiber Layer Thickness According to the operative associations:}

The RNFL thickness in each quadrant was not significantly different by the use of stain assisted ILM peeling (table 12).

The RNFL thickness in each quadrant was not significantly different by combination of PhacoVitrectomy (table 13).

The RNFL thickness in average, nasal, temporal, and superior temporal, inferior nasal, inferior temporal quadrant was changed significantly by use of PFC group (table 14). 
Table (12): Comparison between No (stain-assisted ILM Peel) and Yes (stain-assisted ILM Peel) according to all data in the study group

\begin{tabular}{|c|c|c|c|c|c|c|}
\hline \multirow{2}{*}{ Parameters } & \multicolumn{2}{|c|}{ No ILM Peel } & \multicolumn{2}{c|}{ Yes ILM Peel } & \multicolumn{2}{c|}{ t-test } \\
\cline { 2 - 7 } & Mean & \pm SD & Mean & \pm SD & T & p-value \\
\hline NFL G Change 1w after 1m. & 18.24 & 54.54 & -2.25 & 25.51 & 1.256 & 0.272 \\
\hline NFL G Change 1w after 3m. & 19.44 & 53.18 & -6.43 & 34.27 & 1.942 & 0.174 \\
\hline NFL N Change 1w after 1m. & 9.93 & 44.63 & -10.94 & 21.04 & 1.942 & 0.174 \\
\hline NFL N Change 1w after 3m. & 14.83 & 55.81 & -17.90 & 26.87 & 3.043 & 0.092 \\
\hline NFLT Change 1w after 1m. & 35.59 & 80.65 & 2.51 & 32.09 & 1.538 & 0.225 \\
\hline NFLT Change 1w after 3m. & 39.36 & 85.19 & -0.28 & 58.45 & 1.739 & 0.198 \\
\hline NFL SN Change 1w after 1m. & 8.80 & 72.33 & 4.26 & 35.86 & 0.035 & 0.854 \\
\hline NFL SN Change 1w after 3m. & 13.64 & 76.61 & -4.30 & 35.40 & 0.490 & 0.490 \\
\hline NFL ST Change 1w after 1m. & 75.03 & 219.30 & -2.47 & 24.88 & 1.219 & 0.279 \\
\hline NFL ST Change 1w after 3m. & 96.95 & 303.86 & 7.51 & 56.81 & 0.837 & 0.368 \\
\hline NFL IN Change 1w after 1m. & 22.08 & 72.22 & -1.02 & 8.57 & 0.998 & 0.326 \\
\hline NFL IN Change 1w after 3m. & 13.52 & 64.15 & -2.92 & 19.40 & 0.618 & 0.438 \\
\hline NFL IT Change 1w after 1m. & 30.12 & 106.92 & -8.01 & 19.48 & 1.231 & 0.277 \\
\hline NFL IT Change 1w after 3m. & 21.57 & 86.41 & -20.74 & 23.39 & 2.277 & 0.143 \\
\hline
\end{tabular}

Table (13): Comparison between No Phaco and Yes Phaco according to all data in the study group

\begin{tabular}{|c|c|c|c|c|c|c|}
\hline \multirow{2}{*}{ Parameters } & \multicolumn{2}{|c|}{ No Phaco } & \multicolumn{2}{c|}{ Yes Phaco } & \multicolumn{2}{c|}{ t-test } \\
\cline { 2 - 7 } & Mean & \pm SD & Mean & \pm SD & T & p-value \\
\hline NFL G Change 1w after 1m. & 2.56 & 28.98 & 16.53 & 55.49 & 0.597 & 0.446 \\
\hline NFL G Change 1w after 3m. & 4.55 & 40.42 & 14.44 & 53.62 & 0.280 & 0.601 \\
\hline NFL N Change 1w after 1m. & 1.92 & 34.97 & 3.58 & 42.48 & 0.012 & 0.913 \\
\hline NFL N Change 1w after 3m. & 6.68 & 63.19 & 2.32 & 42.66 & 0.051 & 0.823 \\
\hline NFLT Change 1w after 1m. & 41.75 & 102.12 & 14.62 & 41.32 & 1.063 & 0.311 \\
\hline NFLT Change 1w after 3m. & 38.07 & 99.01 & 19.24 & 66.09 & 0.391 & 0.537 \\
\hline NFL SN Change 1w after 1m. & -6.95 & 41.56 & 15.53 & 70.83 & 0.916 & 0.347 \\
\hline NFL SN Change 1w after 3m. & 3.30 & 54.76 & 10.19 & 72.50 & 0.074 & 0.787 \\
\hline NFL ST Change 1w after 1m. & -3.88 & 47.49 & 79.92 & 222.10 & 1.505 & 0.230 \\
\hline NFL ST Change 1w after 3m. & 10.27 & 69.72 & 100.06 & 310.18 & 0.883 & 0.355 \\
\hline
\end{tabular}




\begin{tabular}{|l|c|c|c|c|c|c|}
\hline NFL IN Change 1w after 1m. & 6.62 & 38.03 & 18.87 & 69.85 & 0.286 & 0.597 \\
\hline NFL IN Change 1w after 3m. & 9.97 & 50.34 & 6.92 & 56.75 & 0.022 & 0.883 \\
\hline NFL IT Change 1w after 1m. & 0.94 & 25.97 & 26.95 & 110.26 & 0.585 & 0.451 \\
\hline NFL IT Change 1w after 3m. & -10.00 & 40.35 & 17.58 & 87.28 & 0.967 & 0.334 \\
\hline
\end{tabular}

Table (14): Comparison between No PFC and Yes PFC according to all data in the study group

\begin{tabular}{|c|c|c|c|c|c|c|}
\hline \multirow{2}{*}{ Parameters } & \multicolumn{2}{|c|}{ No PFC } & \multicolumn{2}{|c|}{ Yes PFC } & \multicolumn{2}{|c|}{ t-test } \\
\hline & Mean & \pm SD & Mean & \pm SD & $\mathbf{T}$ & p-value \\
\hline NFL G Change after $1 \mathrm{w}$ & 111.35 & 28.45 & 86.00 & 29.12 & 5.213 & 0.030 \\
\hline NFL G Change $1 \mathrm{w}$ after $1 \mathrm{~m}$. & 0.88 & 28.38 & 32.47 & 69.30 & 3.183 & 0.085 \\
\hline NFL G Change $1 \mathrm{w}$ after $3 \mathrm{~m}$. & -0.57 & 37.61 & 33.59 & 61.56 & 3.571 & 0.069 \\
\hline NFL N Change after $1 \mathrm{w}$ & 96.15 & 32.25 & 85.30 & 30.00 & 0.789 & 0.382 \\
\hline NFL $\mathrm{N}$ Change $1 \mathrm{w}$ after $1 \mathrm{~m}$. & -8.01 & 23.78 & 24.95 & 54.51 & 5.411 & 0.027 \\
\hline NFL N Change $1 \mathrm{w}$ after $3 \mathrm{~m}$. & -4.78 & 43.74 & 21.31 & 59.57 & 1.860 & 0.183 \\
\hline NfL T Change after $1 \mathrm{w}$ & 99.95 & 26.54 & 71.80 & 28.95 & 7.069 & 0.013 \\
\hline NfL T Change $1 \mathrm{w}$ after $1 \mathrm{~m}$. & 18.55 & 76.84 & 36.61 & 53.52 & 0.441 & 0.512 \\
\hline NfL T Change $1 \mathrm{w}$ after $3 \mathrm{~m}$. & 18.66 & 77.64 & 41.11 & 82.48 & 0.535 & 0.471 \\
\hline NFL SN Change after $1 \mathrm{w}$ & 115.90 & 40.79 & 104.40 & 42.58 & 0.515 & 0.479 \\
\hline NFL SN Change $1 \mathrm{w}$ after $1 \mathrm{~m}$. & 0.20 & 39.54 & 21.46 & 93.32 & 0.780 & 0.385 \\
\hline NFL SN Change $1 \mathrm{w}$ after $3 \mathrm{~m}$. & -3.46 & 48.29 & 29.90 & 90.19 & 1.768 & 0.194 \\
\hline NFL ST Change after $1 \mathrm{w}$ & 134.30 & 33.02 & 91.80 & 53.49 & 7.257 & 0.012 \\
\hline NFL ST Change $1 \mathrm{w}$ after $1 \mathrm{~m}$. & -3.02 & 46.66 & 153.63 & 289.53 & 5.756 & 0.023 \\
\hline NFL ST Change $1 \mathrm{w}$ after $3 \mathrm{~m}$. & 5.54 & 71.76 & 190.33 & 409.76 & 3.962 & 0.056 \\
\hline NFL IN Change after $1 \mathrm{w}$ & 131.00 & 47.17 & 89.90 & 36.46 & 5.813 & 0.023 \\
\hline NFL IN Change $1 \mathrm{w}$ after $1 \mathrm{~m}$. & -5.38 & 17.64 & 53.89 & 90.68 & 8.205 & 0.008 \\
\hline NFL IN Change $1 \mathrm{w}$ after $3 \mathrm{~m}$. & -7.93 & 24.09 & 39.98 & 79.61 & 6.295 & 0.018 \\
\hline NFL IT Change after $1 \mathrm{w}$ & 158.25 & 38.79 & 110.10 & 60.19 & 7.073 & 0.013 \\
\hline NFL IT Change $1 \mathrm{w}$ after $1 \mathrm{~m}$. & -5.87 & 19.85 & 63.96 & 145.41 & 4.602 & 0.041 \\
\hline NFL IT Change $1 \mathrm{w}$ after $3 \mathrm{~m}$. & -9.01 & 27.34 & 40.43 & 119.36 & 3.203 & 0.044 \\
\hline
\end{tabular}

Changes in Retinal Nerve Fiber Layer Thickness According to the associated systemic diseases:

The RNFL thickness in each quadrant was not significantly different by systemic diseases groups. 
Table (15): Relation between systemic Diseases according to all data in the study group

\begin{tabular}{|c|c|c|c|c|c|c|c|c|}
\hline \multirow{3}{*}{ Parameters } & \multicolumn{6}{|c|}{ Systemic D } & \multirow{2}{*}{\multicolumn{2}{|c|}{ ANOVA }} \\
\hline & \multicolumn{2}{|c|}{ No } & \multicolumn{2}{|c|}{ Diabetes } & \multicolumn{2}{|c|}{ Diabetes+Hypertension } & & \\
\hline & Mean & $\pm \mathrm{SD}$ & Mean & \pm SD & Mean & \pm SD & $\mathbf{F}$ & $\begin{array}{c}\text { p- } \\
\text { value }\end{array}$ \\
\hline $\begin{array}{l}\text { NFL G Change } 1 \mathrm{w} \\
\text { after } 1 \mathrm{~m} .\end{array}$ & 15.97 & 58.41 & 13.48 & 28.14 & 2.98 & 47.11 & 0.198 & 0.821 \\
\hline $\begin{array}{l}\text { NFL G Change } 1 \mathrm{w} \\
\text { after } 3 \mathrm{~m} .\end{array}$ & 11.05 & 53.55 & 17.66 & 39.51 & 4.39 & 53.25 & 0.148 & 0.863 \\
\hline $\begin{array}{l}\text { NFL N Change } 1 \mathrm{w} \\
\text { after } 1 \mathrm{~m} .\end{array}$ & 11.12 & 47.35 & 7.22 & 15.13 & -12.57 & 40.37 & 1.034 & 0.369 \\
\hline $\begin{array}{l}\text { NFL N Change } 1 \mathrm{w} \\
\text { after } 3 \mathrm{~m} .\end{array}$ & 2.90 & 54.08 & 18.23 & 53.61 & -7.33 & 43.04 & 0.538 & 0.590 \\
\hline $\begin{array}{l}\text { NFLT Change } 1 \mathrm{w} \\
\text { after } 1 \mathrm{~m} .\end{array}$ & 24.04 & 41.37 & 43.65 & 119.58 & 8.37 & 42.18 & 0.528 & 0.596 \\
\hline $\begin{array}{l}\text { NFLT Change } 1 \mathrm{w} \\
\text { after } 3 \mathrm{~m} .\end{array}$ & 24.62 & 70.71 & 36.71 & 106.93 & 18.95 & 68.63 & 0.106 & 0.900 \\
\hline $\begin{array}{c}\text { NFL SN Change } 1 \mathrm{w} \\
\text { after } 1 \mathrm{~m} .\end{array}$ & 4.71 & 86.17 & 15.20 & 35.31 & 3.98 & 39.64 & 0.084 & 0.920 \\
\hline $\begin{array}{c}\text { NFL SN Change } 1 \mathrm{w} \\
\text { after } 3 \mathrm{~m} .\end{array}$ & 1.31 & 81.28 & 27.45 & 63.47 & -0.74 & 40.55 & 0.480 & 0.624 \\
\hline $\begin{array}{l}\text { NFL ST Change } 1 \mathrm{w} \\
\text { after } 1 \mathrm{~m} .\end{array}$ & 83.72 & 257.33 & 16.83 & 56.04 & 28.10 & 119.84 & 0.404 & 0.672 \\
\hline $\begin{array}{c}\text { NFL ST Change } 1 \mathrm{w} \\
\text { after } 3 \mathrm{~m} .\end{array}$ & 109.64 & 370.34 & 37.52 & 77.34 & 32.07 & 115.83 & 0.313 & 0.734 \\
\hline $\begin{array}{l}\text { NFL IN Change } 1 \mathrm{w} \\
\text { after } 1 \mathrm{~m} .\end{array}$ & 19.96 & 67.05 & 3.23 & 16.46 & 16.22 & 75.88 & 0.190 & 0.828 \\
\hline $\begin{array}{l}\text { NFL IN Change } 1 \mathrm{w} \\
\text { after } 3 \mathrm{~m} .\end{array}$ & 14.97 & 61.12 & 1.02 & 23.37 & 4.26 & 64.79 & 0.188 & 0.830 \\
\hline $\begin{array}{l}\text { NFL IT Change } 1 \mathrm{w} \\
\text { after } 1 \mathrm{~m} .\end{array}$ & 21.84 & 96.59 & -4.33 & 19.60 & 30.33 & 117.31 & 0.333 & 0.720 \\
\hline NFL IT Change $1 \mathrm{w}$ & 14.40 & 81.44 & 3.17 & 30.14 & 1.29 & 94.63 & 0.096 & 0.909 \\
\hline
\end{tabular}


after $3 \mathrm{~m}$.

Correlation between changes in RNFL thickness and Visual Acuity and IOP:

Changes in RNFL thickness was highly significantly correlated to visual acuity and IOP.

Table (16): The extent of the difference over the periods through visual acuity in the study group

\begin{tabular}{|c|c|c|c|c|c|}
\hline \multirow{2}{*}{ Period } & \multicolumn{2}{|c|}{ Visual Acuity } & \multicolumn{3}{c|}{ Paired Sample t-test } \\
\cline { 2 - 6 } & Range & Mean \pm SD & Mean Diff. & t-test & p-value \\
\hline After 1wk. & $0.001-0.004$ & $0.002 \pm 0.001$ & & & \\
\hline After 1m. & $0.004-0.03$ & $0.010 \pm 0.009$ & 0.01 & -4.623 & $<0.001^{* *}$ \\
\hline After 3m. & $0.004-0.3$ & $0.084 \pm 0.099$ & 0.08 & -4.558 & $<0.001^{* *}$ \\
\hline
\end{tabular}

**p-value $<0.001 \mathrm{HS}$

Table (17): The extent of the difference over the periods through IOP in the study group

\begin{tabular}{|c|c|c|c|c|c|}
\hline \multirow{2}{*}{ Period } & \multicolumn{2}{|c|}{ IOP } & \multicolumn{3}{c|}{ Paired Sample t-test } \\
\cline { 2 - 6 } & Range & Mean \pm SD & Mean Diff. & t-test & p-value \\
\hline After 1wk. & $18-24$ & $20.83 \pm 1.82$ & & & \\
\hline After 1m. & $16-23$ & $19.63 \pm 2.09$ & -1.20 & 3.714 & $<0.001^{* *}$ \\
\hline After 3m. & $13-23$ & $18.77 \pm 2.58$ & -2.06 & 4.520 & $<0.001^{* *}$ \\
\hline
\end{tabular}

**p-value $<0.001 \mathrm{HS}$

\section{Discussion:}

The number of retinal ganglion cells in the normal adult ranges from 700,000 to 1.4 million, and it decreases with aging. The human RNFL loses 5,000 axons per year from birth to death, 2,500 per year before age 50, and 7,500 per year after age $50^{(1,4)}$.

Central or peripheral visual field defects can occur as complications of vitrectomy. The pathomechanism of visual field defects after vitrectomy, however, is under debate and various hypotheses have been considered.

There are several possible explanations: a postoperative increase in IOP, retinal toxicity of intravitreal gas tamponade, phototoxicity from endoillumination, mechanical retinal damage during posterior vitreous peeling, 
mechanical damage of nerve fiber layer in ERM or ILM peeling, dehydration of the RNFL caused by fluid-air exchange, and chemical retinal damage caused by ICG stain ${ }^{(5)}$.

Kerrigan-Baumrind et al. ${ }^{(6)}$ reported that at least $25 \%$ to $35 \%$ retinal ganglion cell loss is associated with statistical abnormalities in automated visual field testing.

The RNFL thickness measured by SDOCT objectively and qualitatively is important as RNFL damage often occurs earlier before visual field defects and $\mathrm{ONH}$ damage can be detected.

Retinal ganglion cell death can be induced by retinal ischemia. Retinal ganglion cells are particularly sensitive to acute, transient, and mild systemic hypoxic stress ${ }^{(4)}$.

In retinal vessels, a superficial retinal vessel layer exists within the ganglion cell layer and nerve fiber layer, and a deep retinal vessel layer exists within the inner nuclear layer.

Pichi et al. (2), found that there were frequent postoperative inner retina layer modifications in vitrectomy cases.

The present study included 30 patients who underwent pars plana vitrectomy combined by silicon endotamponade, we found that the changes of RNFL did not change significantly along the 3 months follow up in all quadrants. Lee et al. ${ }^{(7)}$ and
KIM et al. ${ }^{(8)}$ also found that there are no changes in the RNFL after vitrectomy.

This study found that changes of RNFL did not differ significantly with the age of the patient or their gender type, Takkar et al. ${ }^{(9)}$ also found that there are no statistically significant changes of RNFL in correlation with the patient's age except at the temporal quadrant.

In the present study we found that the RNFL is significantly changed in correlation with axial length at almost all quadrants at the 3 months follow up, this is similar to Chen et al. (10) who found that there are significant correlation between RNFL thickness and axial length in non-temporal area, in contrast to Takkar et al. ${ }^{(9)}$ who did not find significant changes with axial length of the eye.

From our data we found that the RNFL thickness in each quadrant was not significantly different by pathology group, Kim et al. ${ }^{(8)}$ found also that RNFL thickness in each quadrant of either the fellow eyes or vitrectomized eyes was not significantly different by disease group.

In our study we found that no statistically significant difference in RNFL thickness in correlation to presence or absence of systemic diseases, this was matched with Minami et al. ${ }^{(11)}$.

The ILM, a thin membrane in the innermost layer of the retina formed by the 
footplates of muller cells, is an anatomical boundary between the retina and vitreous.

Wolf et al. ${ }^{(12)}$ reported that substantial damage to Muller cell end feet is caused by ILM peeling, even in the untouched retina directly adjacent to the peeled area. This suggests that the basal lamina can transmit mechanical forces to the inner retinal layers such that cellular damage occurs in these layers.

Yamashita et al. ${ }^{(5)}$ assessed the RNFL after ICG-assisted vitrectomy and found that the RNFL was significantly reduced postoperatively. Haritoglou et al. ${ }^{(13)}$ reported central scotoma in 59 of 105 cases $(56.2 \%)$ after ILM peeling in patients with $\mathrm{MH}$ and presumed that direct mechanical injury to the RNFL during ILM peeling caused visual field defects. Furthermore, Kim et al. ${ }^{(8)}$ observed a significant decrease in the RNFL thickness in the superior quadrant after vitrectomy with ICG-assisted ILM peeling compared with the preoperative measurements.

Postoperative thinning of the temporal RNFL is explained by the recovery from transitory RNFL swelling caused by traction forces during ILM peeling, which causes increased RNFL thickness and retinal edema was diminished over time ${ }^{(7)}$.

Paradoxically, thickening was also reported, it was found that the average and temporal RNFL thicknesses of affected eyes were thicker than those of unaffected fellow eyes, this is explained by the Disc edema which was caused by postoperative inflammation which might have affected the RNFL thickness ${ }^{(7)}$.

In the present study our findings were not consistent with the other authors as we observed no statistically significant differences in changes of RNFL thickness in correlation with stain-assisted ILM peeling. Our findings however, matched those of Toba et al. ${ }^{(14)}$ who found that the degree of change of the RNFL thickness was not significantly related to the type of vital stain (Triamcinolone acetanoid, Brilliant blue, trypan blue and ICG) used during surgery.

Triamcinolone acetonide (TA) is a steroidal compound that has also been used to make the posterior vitreous membrane and ILM more visible Kumagai et al. ${ }^{(15)}$. TA is not soluble in the vitreous, and the presence of TA particles on the retinal surface enables surgeons to see where the ILM has been peeled as the area lacking white specks. TA is used in all cases enrolled in this study and we did not noticed any significant changes of RNFL thickness which resembles Toba et al. ${ }^{(14)}$ study, who did not find any significant correlation between the TA use and changes of RNFL thickness.

This study demonstrated that the RNFL thickness there was not statistically significantly different in correlation with combined phacovitrectomy procedure, which is consistent with Gharbiya and Lyssek-Boroń who did not notice 
any differences in the RNFL thickness between patients who underwent phacovitrectomy and those who underwent vitrectomy alone. Conversely Lalezary found that Phacovitrectomy may contribute to significant increases in the RNFL thicknesses ${ }^{(16,17)}$.

Perfluorocarbon liquids (PFCLs) are useful and safe surgical tools in vitreoretinal surgery. The use of PFCL as a tamponade has been controversial due to the corneal toxicity, retinal infiltration, and inflammatory reaction in experimental studies. When PFCLs are used as postoperative tamponades for more than one week, a foreign-body inflammatory reaction is observed in up to $30 \%$ of cases but such a reaction does not induce PVR, and it resolves after removal of PFCLs. Although most clinical studies have found no signs of retinal toxicity such as progressive visual acuity deterioration, few performed ERG or retinal histological analysis ${ }^{(18)}$.

Because of the lack of reports in the literatures, we are surprised by the result of PFC on changes of RNFL thickness which was statistically significant. We noticed that correlation was significant at the first week in (global, nasal, superior temporal and inferior temporal). We observed this significance also at the $3^{\text {rd }}$ month as a thinning in only (inferior nasal and inferior temporal).

We hypothesize that the significant correlation related to thickening of RNFL due to rebound edema caused by compression by heavy liquid like PFC, while the reported correlation related to thinning of RNFL is caused by toxic effect of the liquid itself, also both thinning or thickening may be explained by inaccurate segmentation by OCT device or sampling bias.

Silicone oil-based endotamponade is a common substance used as a long-term tamponade. It has hydrophobic nature and helps retinal tears to be sealed.

In our study we found that there are no statistically significant changes in RNFL due to use of silicon endotamponade, also this was observed by Caramoy et al. ${ }^{(19)}$. Although silicone oils have been used for years and are relatively safe (which matches with our study), there are cases of RNFL thinning after the use of silicone oil ${ }^{(9)}$.

In contrast to Darija et al. (20) who achieved vitrectomy for 47 patients with Retinal detachment and silicone oil endotamponade for 6 months. They found thickening of RNFL in vitrectomized eyes during silicon tamponade and after removal.

All of the cases of our study underwent Endolaser retinal photocoagulation and our statistical analysis showed no significant correlation between PRP and changes of RNFL thickness which matched results of Kim and Cho ${ }^{(21)}$, who proved that although a decrease in peripapillary RNFL thickness was observed in the treatment group after 6 months, it was not statistically significant compared to control group. 
This was Dissimilar to results of Eren et al. ${ }^{(22)}$ who used PRP for 58 eyes with PDR and followed them up for 6 months, they reported both increase and decrease in RNFL thickness giving us the following explanations; RNFL Thickness increase in the third month of follow-up may be related to ensuing axonal edema. Significant RNLF thickness decrease at the sixth month of follow-up may be attributed to axonal loss secondary to the laser treatment.

Also it was reported that, diabetic eyes that have been treated with PRP have thinner RNFL than non-diabetic eyes. Optic nerves in eyes treated with PRP are more likely to be graded as abnormal, but their appearance is not necessarily glaucomatous and may be related to thinning of the RNFL ${ }^{(23)}$.

We reported that the visual acuity was improved significantly, which was statistically significant correlated with the changes of RNFL. We explained that improvement was due to repair of the original pathology.

This study demonstrated that the IOP is statistically significant decreased along the period of follow up.

\section{Conclusion:}

In conclusion, RNFL tends to change post vitrectomy but not significantly, careful examination and consistent follow-up are required for vitrectomized patients.

Retinal damage related to vitrectomy can be minimized by increasing the accuracy of the surgeon performing the techniques, controlling IOP during surgery, decreasing the surgical duration, and using an endoilluminator with specific wavelength.

In the postoperative followup, the SDOCT can be used as an objective tool for evaluating RNFL thickness after vitrectomy, and large and long-term prospective studies are needed to evaluate changes in the RNFL after vitrectomy by SD-OCT.

Histopathologic evaluation of the retinal microcirculation and the degree of retinal damage are required to determine the mechanism of retinal damages that might occur during vitrectomy.

\section{References:}

1. Quigley HA, Dunkelberger GR, Green WR. Retinal ganglion cell atrophy correlated with automated perimetry in human eyes with glaucoma. Am J Ophthalmol 1989; 107: 453-464.

2. Pichi F, Lembo A, Morara M. Early and late inner retinal changes after inner limiting membrane peeling. Int Ophthalmol., 2014; 34: 437-446. 
3. Romano MR, Angi M, Valldeperas X, Costagliola C, Vinciguerra P. Twenty- three- gauge pars plana vitrectomy, and $360^{\circ}$ endolaser versus combined 20- gauge pars plana vitrectomy, scleral buckle, and SF6 for pseudophakic retinal detachment with inferior retinal breaks. Retina 2011; 31: 686- 91.

4. Budenz DL, Anderson DR, Varma R, Schuman J, Cantor L, Savell J. Determinants of normal retinal nerve fiber layer thickness measured by Stratus OCT. Ophthalmology 2007; 114: 1046-52.

5. Yamashita $\mathrm{T}$, Uemura A, Kita $\mathrm{H}$, Sakamoto T. Analysis of the retinal nerve fiber layer after indocyanine green-assisted vitrectomy for idiopathic macular holes. Ophthalmology 2006; 113: 280-284.

6. Kerrigan-Baumrind LA, Quigley HA, Pease ME. Number of ganglion cells in glaucoma eyes compared with threshold visual field tests in the same persons. Invest Ophthalmol Vis Sci 2000; 41: 741-748.

7. Lee SB, Shin YI, Jo YJ, Kim JY. Longitudinal changes in retinal nerve fiber layer thickness after vitrectomy for epiretinal membrane. Invest Ophthalmol Vis Sci. 2014; 55: 6607-6611.

8. Kim KY, Yu SY, Kim MS, Kim ES, Kwak HW. Changes of parafoveal retinal nerve fiber layer thickness analyzed by spectral-domain optical coherence tomography after pars plana vitrectomy. Retina. 2013; 33(4): 776-84.

9. Takkar B, Azad R, Kamble N, Azad S. Retinal nerve fiber layer changes following primary retinal detachment repair with silicone oil tamponade and subsequent oil removal. J Ophthalmic Vis Res 2018; 13: 124-9.

10. Chen CY, Huang EJC, Kuo CN, Wu PL, Chen CL, $\mathrm{Wu}$ PC. The relationship between age, axial length and retinal nerve fiber layer thickness in the normal elderly population in Taiwan: The Chiayi eye study in Taiwan. PLoS ONE, 2018; 13(3): e0194116.

11. Minami M, Oku H, Okuno T, Fukuhara M, Ikeda T. High infusion pressure in conjunction with vitreous surgery alters the morphology and function of the retina of rabbits. Acta Ophthalmologica Scandinavica. 2007; 85(6): 633-9.

12. Wolf S, Schnurbusch U, Wiedemann P, Grosche J, Reichenbach A, Wolburg H. Peeling of the basal membrane in the human retina: ultrastructural effects. Ophthalmology. 2004; 111: 238-243.

13. Haritoglou C, Gass CA, Schaumberger M. Macular changes after peeling of the internal limiting membrane in macular hole surgery. Am J Ophthalmol. 2001; 132: 363-368.

14. Toba Y, Machida S, Kurosaka D. Comparisons of retinal nerve fiber layer thickness after indocyanine green, 
brilliant blue $\mathrm{g}$, or triamcinolone acetonide-assisted macular hole surgery. Journal of Ophthalmology, 2014; 187308.

15. Kumagai K, Furukawa M, Ogino N, Larson E, Uemura A. Long-term outcomes of macular hole surgery with triamcinolone acetonide-assisted internal limiting membrane peeling. Retina, 2007; 27(9): 1249-1254.

16. Gharbiya M, La Cava M, Tortorella P. Peripapillary RNFL thickness changes evaluated with spectral domain optical coherence tomography after uncomplicated macular surgery for epiretinal membrane. Seminars in Ophthalmology, 2016; 1-7.

17. Lyssek-Boroń A, Wylęgała A, Polanowska K, Krysik K, Dobrowolski D. Longitudinal changes in retinal nerve fiber layer thickness evaluated using avanti Rtvue-XR optical coherence tomography after $23 \mathrm{G}$ vitrectomy for epiretinal membrane in patients with open-angle glaucoma. Journal of Healthcare Engineering. 2017; 2017.

18. Figueroa MS, Casas DR. Inflammation induced by perfluorocarbon liquid: intraand postoperative use. BioMed Research International. 2014; 2014.

19. Caramoy A, Droege KM, Kirchhof B, Fauser S. Retinal layers measurements in healthy eyes and in eyes receiving silicone oil- based endotamponade. Acta Ophthalmologica. 2014; 92(4): e292-7.

20. Darija J, Mia ZG, Ivan Ć, Dobrila KU. Retinal Layers Measurements following Silicone Oil Tamponade for Retinal Detachment Surgery, Seminars in Ophthalmology, 2017.

21. Kim HY, Cho HK. Peripapillary retinal nerve fiber layer thickness change after panretinal photocoagulation in patients with diabetic retinopathy. Korean Journal of Ophthalmology. 2009; 23(1): 23-6.

22. Eren S, Ozturk T, Yaman A, Oner H, Osman SA. Retinal nerve fiber layer alterations after photocoagulation: a prospective spectral-domain OCT study. The Open Ophthalmology Journal. 2014; 8: 82 .

23. Lim MC, Tanimoto SA, Furlani BA, Lum B, Pinto LM, Eliason D, Prata TS, Brandt JD, Morse LS, Park SS, Melo LA. Effect of diabetic retinopathy and panretinal photocoagulation on retinal nerve fiber layer and optic nerve appearance. Archives of Ophthalmology. 2009; 127(7): 857-62. 